\title{
Body fat fluctuations among female adolescents with restrained eating behaviours
}

\author{
Zhaoxiu Lai \\ Yong Peng Why \\ David Soo Quee Koh \\ Vivian Ah Ching Ng \\ Chin Leong Lim
}

\begin{abstract}
Body fat fluctuation during varying competing academic demand was examined among female adolescents with high $(n=24)$ or low $(n=24)$ restrained eating behaviours. Body fat, perceived stress, energy intake, and exercise behaviour were measured three times with 10 weeks between consecutive sessions. High restrained eaters' body fat increased during a period when academic demands were high. Significant results were found for exercise behaviours but not for food consumption. Post hoc analyses did not reveal significant results for low restrained eaters. The results lend support to Muraven and Baumeister's limited strength model of self-regulation.
\end{abstract}

Keywords

Stress

Obesity

Eating behaviours

Restrained eating

Female

Body fat

Exercise

(C) 2012, Elsevier. Licensed under the Creative Commons Attribution-NonCommercialNoDerivatives 4.0 International http://creativecommons.org/licenses/by-nc-nd/4.0/ 


\section{Introduction}

Obesity is fast becoming an epidemic in many industrialised nations. Not only does obesity pose a heavy economic burden, it also confers an increased risk in diabetes, heart disease, hypertension, other metabolic disorders, and mortality (Stein \& Colditz, 2004). Research into the psychosocial aspects of body fat gain has been actively pursued in health psychology. Presently, a group of individuals who chronically engage in dieting behaviours, or restrained eating behaviours, have been identified as a group who is potentially at risk for obesity (van Strien, Engels, van Staveren, \& Herman, 2006).

It is controversial whether individuals who engage in restrained eating behaviours are at a higher risk of obesity. While some studies have reported that individuals with restrained eating behaviours tend to increase food consumption during high psychological stress (Zellner et al., 2006), other studies have also reported null results (Lowe et al., 2006). It is important to consider that a gain in body fat requires a positive energy balance, which may entail either an increased energy intake or a decreased energy expenditure.

The regulation of energy intake and expenditure is particularly taxed during high psychological stress given that self-regulatory resources are limited. Self-regulation, a process by which an individual overcomes goal-irrelevant impulses (e.g., bingeing) and changes behavioural responses in order to attain one's goal or ideal (e.g., losing weight), has been found to function based on a limited resource that can be depleted to result in selfregulatory failure (Muraven \& Baumeister, 2000). That is, it might be difficult for an individual with restrained eating behaviours to simultaneously self-regulate their energy intake, energy expenditure, and cope with their stressors competently. A number of studies have found that cognitively taxing tasks tend to increase food consumption among individuals with restrained eating behaviours (van Strien et al., 2006), which suggests that the restriction of energy intake is cognitively demanding. Physical exercise also taxes selfregulatory resources (Sonnentag \& Jelden, 2009). We hypothesise that the occurrence of selfregulatory failure can fluctuate due to a change in the number of competing activities, which might result in an increase in body fat percentage when competing demands are high and a decrease in body fat percentage when competing demands are low. Such fluctuations in body weight have been found to increase one's risk of cardiovascular disease and mortality (Montani, Viecelli, Prevot, \& Dulloo, 2006). However, the assessment of both energy intake and expenditure among individuals with restrained eating behaviours to explain for their body fat changes during psychological stress in a naturalistic setting has often not been done.

\section{Method}

\section{Participants}

Three-hundred and five Chinese female first year undergraduates were administered the Dutch Eating Behaviour Questionnaire Restrained Eating scale (DEBQ-R; van Strien, Frijters, Bergers, \& Defares, 1986). A homogenous sample was taken in terms of gender and ethnicity because restrained eating behaviour has been found to be more relevant for women (Kiefer, Rathmanner, \& Kunze, 2005) and ethnic differences in the country tend to covary with religious dietary habits (e.g., fasting among Muslim participants during Ramadan), which might be problematic for this five month prospective study. 
Individuals with high and low restrained eating behaviours were identified as $1 S D$ above and below the mean respectively for the DEBQ-R. From 305 participants, a total of 114 participants were identified. The 114 individuals were contacted via phone and screened further for eligibility. Individuals who scored 16 and above on the Beck's Depression Inventory ( $N=14$; Beck, Ward, Mendelson, Mock, \& Erbaugh, 1961) or had restricted dietary patterns $(N=4$; e.g., vegans) were deemed ineligible for this study. None of the participants reported having any eating (e.g., anorexia), endocrine (e.g., hyperthyroidism) or metabolic (e.g., diabetes) disorders. Fifty-six participants out of the 114 came for a briefing and eventually 50 participants volunteered for this study. One participant dropped out of the study midway while another participant had substantial missing data. Hence, complete data for 48 participants was available and analysed. Of the 48 participants, 24 were identified as individuals with high restrained eating behaviours and 24 were those with low restrained eating behaviours. Mean age of the sample was 19.19 years $(S D=0.67)$ and mean BMI was $20.74 \mathrm{~kg} / \mathrm{m}^{2}(S D=3.25)$.

\section{Measures}

The DEBQ-R was used to identify those high and low in restrained eating behaviours. This scale has been found to be reliable and valid (van Strien et al., 1986). Cronbach alpha for this scale in the current study was .91. Perceived stress was assessed using the Perceived Stress Scale (PSS; Cohen, Kamarck, \& Mermelstein, 1983). The scale was modified for this study to assess perceived stress within the last 1 week from the time of assessment. PSS was administered at each of the three time points. Cronbach alphas for this scale ranged from .84 to .88 .

Body fat percentage (\%BF) of the participants was estimated using skinfold measurements taken with Harpenden skinfold calipers (British Indicators, UK). Three trained female observers took participants' measurements at the triceps, subscapular, biceps, and suprailiac sites to the nearest $0.1 \mathrm{~mm}$. The measurements were taken according to the protocol of the International Society for the Advancement of Kinanthropometry (Marfell-Jones, Olds, Stewart, \& Carter, 2006). The sum of the skinfolds was entered into Durnin and Womersley's (1974) equation for females to predict body density. This equation has been validated among Singaporean Chinese females (Deurenberg \& Deurenberg-Yap, 2002). The equation (4.84/body density) - 4.37 (Heyward \& Wagner, 2004; p. 9) was used to estimate \%BF because it minimises systematic errors in predicting the \%BF of Singaporeans. Following the suggested guidelines (Norton \& Olds, 2002; p. 395-421), the observers collected precision data using 10 female volunteers. The average intraobserver technical error of measurement (TEM) was $3.26 \%$, within the acceptable limit of 5\%. The interobserver TEM was $4.15 \%$, within the recommended target of $7.5 \%$.

Two factors that contribute to body fat changes measured in this study were energy intake via the standard 3-day food diary and energy expenditure via physical exercise. Using a standardised food diary (Low, 2009), participants recorded their dietary information over two weekdays and one weekend day. To determine the amount of energy intake per day, participants' records were analysed using the Food Intake Assessment programme, which has the caloric density of local food items (Centre for Molecular Epidemiology, 2010). For energy expenditure, Godin's Leisure Time Exercise Questionnaire (GLTEQ; Godin \& Shephard, 1985) was used to measure participants' level of weekly exercise.

\section{Procedure}


This study utilised a prospective design with three time points separated by 10 -week intervals. The baseline took place at the beginning of a new academic semester (August or January) when competing academic demands were low. The pre-examination time point occurred 3 weeks before examinations (November or April) when competing academic demands were higher and the recovery point occurred during vacation (January or June) when competing academic demands were at its lowest. Hence, participants were sampled in one of the two temporal waves (i.e., August-November-January or January-April-June).

Within 1 week after the food diary recordings, participants attended individually conducted laboratory sessions. Seven trained female interviewers verified the food diary records with the participants using a standardised interview protocol (Low \& Lai, 2010). Interobserver deviation was minimised through adherence to the protocol and discussions with the main experimenters to resolve ambiguity that arose from the interviews. Participants also completed the PSS and GLTEQ which were administered in a counterbalanced order. Skinfold measurements were then taken. As far as possible, interviewers and observers were assigned to the same participant throughout the study. Participants' perceived stress levels, energy intake, weekly exercise, and skinfolds were measured at each of the three time points following the procedure outlined here.

\section{Statistical analysis}

Alpha was set at .05. MANOVAs with multivariate repeated measures were conducted with restrained eating group as the two-level between-subject variable (high versus low), and time as the within-subject variable (baseline, pre-examination, recovery) for the dependent variables: \%BF, energy intake, and energy expenditure (physical exercise).

\section{Results}

\section{Perceived stress}

To assess whether the three time points reflected the low-high-low stress cycle within the participants' academic term, a MANOVA was conducted with PSS as the dependent variable. As predicted, the main effect for time was significant, Wilks' $\Lambda=.87, F(2,45)=3.52$, $p=.038$, partial $\eta^{2}=.14$. Post hoc comparisons found that PSS was not significantly different between baseline and pre-examination, $F(1,47)=3.25, p=.078$, partial $\eta^{2}=.07$, but was significantly different between pre-examination and recovery, $F(1,47)=6.45, p=.014$, partial $\eta^{2}=.12$. Mean $(S D)$ PSS for baseline, pre-examination, and recovery are as follows: 15.31(5.96), 16.65(5.73), 14.54(5.56). The main effect for restrained eating group was also significant, $F(1,46)=5.59, p=.02$, partial $\eta^{2}=.11$. Individuals with high restrained eating behaviours tend to report higher PSS, $\mathrm{M}(S D)=17.00(4.66)$, than those with low restrained eating behaviours, $\mathrm{M}(S D)=14.00(4.55)$. The restrained eating by time interaction term was non-significant, Wilks' $\Lambda=.92, F(2,45)=1.84, p=.17$, partial $\eta^{2}=.08$.

\section{Body fat percentage}

The main effect for restrained eating group was significant, $F(1,46)=8.73, p=.005$, partial $\eta^{2}=.16$ : individuals with high restrained eating behaviours had higher $\% \mathrm{BF}$ than those with low restrained eating behaviours (Table 1). Time did not have an effect on \%BF, Wilks' $\Lambda=0.90, F(2,45)=2.58, p=.087$, partial $\eta^{2}=.10$. The restrained eating group by time interaction term was also significant, Wilks' $\Lambda=0.72, F(2,45)=8.59, p=.001$, partial 
$\eta^{2}=.28$. Post hoc analysis indicated that individuals with high restrained eating behaviours' \%BF increased significantly between baseline and pre-examination, $F(1$, $23)=7.45, p=.012$, partial $\eta^{2}=.25$, and decreased significantly between pre-examination and recovery, $F(1,23)=14.85, p=.001$, partial $\eta^{2}=.39$ (Table 1). Since individuals with high restrained eating behaviours showed an increase in \% BF during pre-examination period, it was appropriate to examine if this pattern was replicated in terms of a positive energy balance via energy intake and energy expenditure (physical exercise).

\section{Energy intake}

The time main effect was not significant, Wilk's $\Lambda=0.89, F(2,45)=2.72, p=.076$, partial $\eta^{2}=.11$. The main effect for restrained eating group was significant, $F(1,46)=6.36$, $p=.015$, partial $\eta^{2}=.12$; individuals with high restrained eating behaviours had a lower energy intake compared to those with low restrained eating behaviours (Table 1). The restrained eating group by time interaction was not significant, Wilks' $\Lambda=0.99, F(2$, 45) $=0.26, p=.77$, partial $\eta^{2}=.01$.

\section{Energy expenditure (physical exercise)}

Restrained eating group main effect was not significant, $F(1,46)=0.62, p=.44$, partial $\eta^{2}=.01$. The main effect for time was significant, Wilks' $\Lambda=0.83, F(2,45)=4.62, p=.015$, partial $\eta^{2}=.17$. However, this time main effect has to be interpreted within the context of its interaction with Restrained eating group because it was not significant among the low restrained eating group, Wilks' $\Lambda=0.95, F(2,22)=0.61, p=.55$, partial $\eta^{2}=.05$. The restrained eating group by time interaction term was significant, Wilks' $\Lambda=0.81, F(2$, $45)=5.15, p=.01$, partial $\eta^{2}=.19$. Only individuals with high restrained eating behaviours showed a significant decrease in exercise level between baseline and pre-examination period, $F(1,23)=6.78, p=.016$, partial $\eta^{2}=.23$, and a significant increase during recovery, $F(1$, $23)=14.34, p=.001$, partial $\eta^{2}=.38$ (Table 1).

\section{Discussion}

The current study found that female adolescents high in restrained eating behaviours have significant changes in their body fat percentage over a 20 -week period. These changes in their body fat percentage are likely to be due to their lowered energy expenditure via physical exercise. Hence, individuals with restrained eating behaviours could be one of the high risk groups who might experience a higher likelihood of body fat increase when they fail to selfregulate their body fat levels via physical exercise amidst competing demands that might strain their self-regulatory resources (Muraven \& Baumeister, 2000).

Multiple stressors might strain limited self-regulatory resources and result in a failure in coping with stress. There is some evidence that individuals with restrained eating behaviours increased their food consumption after engaging in a conflict situation that taps into their selfregulatory resources (van Strien et al., 2006). However, even though individuals with restrained eating behaviours can fail to self-regulate their eating behaviours, eating is one aspect of human behaviour that is consistently regulated and hence, may be resistant to selfregulatory failure owing to practice (Muraven \& Baumeister, 2000). Null research findings have led some researchers to question the validity of the restrained eating construct (Stice, Fisher, \& Lowe, 2004). Our results are supportive of this construct rather than refuting: individuals with restrained eating behaviours were found to generally consume fewer calories 
than individuals with less restrained eating behaviours. In addition, such effects are usually manifested when an individual's self-regulatory resources are taxed by other competing demands, as in the pre-examination period in our study (van Strien et al., 2006). The resulting fluctuating energy balance due to self-regulatory failure of either food intake or physical exercise could explain for the changes in body fat percentage of those individuals with restrained eating behaviours. Such weight fluctuations increase one's risk of cardiovascular disease and mortality (Montani et al., 2006).

Although the body fat percentage fluctuations among individuals with restrained eating behaviours were small- $0.56 \%$ and $0.69 \%$ changes between the baseline, pre-examination and the recovery period - such small changes in anthropometric characteristics such as body weight and body fat percentage have been found to have important clinical implication for obesity; a follow-up of the National Health and Nutrition Examination Survey (NHANES) between 1974 and 1980 to 1988 and 1994 (9-18 years follow-up) found that the mean BMI among women in the 20-29 years old age group, a group similar to our participants, had a $4.76 \%$ increase in BMI, which translates to an approximately $0.26-0.53 \%$ annual increase in BMI. This small annual increase in BMI for this subsample had a $64 \%$ increase in obesity prevalence at the follow-up (Flegal \& Troiano, 2000).

To conclude, our study found that individuals with high restrained eating behaviours display significant fluctuations of body fat percentage. The increase in body fat percentage among these individuals appears to occur during a period of high competing activities that strained their self-regulation of energy balance via self-regulatory failure for physical exercise. Weight fluctuations have been found to predict cardiovascular disease and mortality. Future studies might examine whether repeated practice for self-control in maintaining energy balance when competing demands are high might be helpful in improving the self-regulatory strength of these individuals. 


\section{References}

Beck et al., 1961

A.T. Beck, C.H. Ward, M. Mendelson, J. Mock, J. Erbaugh

An inventory for measuring depression

Archives of General Psychiatry, 4 (1961), pp. 561-571

Centre for Molecular Epidemiology, 2010

Centre for Molecular Epidemiology, National University of Singapore (2010). Food intake assessment. [Computer software]. Retrieved from <http://www.nus-cme.org.sg/efood_PSH/>.

Cohen et al., 1983

S. Cohen, T.W. Kamarck, R. Mermelstein

A global measure of perceived stress

Journal of Health and Social Behavior, 24 (1983), pp. 385-396

Deurenberg and Deurenberg-Yap, 2002

P. Deurenberg, M. Deurenberg-Yap

Validation of skinfold thickness and hand-held impedance measurements for estimation of body fat percentage among Singaporean Chinese, Malay and Indian subjects

Asia Pacific Journal of Clinical Nutrition, 11 (2002), pp. 1-7

Durnin and Womersley, 1974

J.V.G.A. Durnin, J. Womersley

Body fat assessed from total body density and its estimation from skinfold thickness.

Measurements on 481 men and women aged from 16 to 72 years

British Journal of Nutrition, 32 (1974), pp. 77-97

Flegal and Troiano, 2000

K.M. Flegal, R.P. Troiano

Changes in the distribution of body mass index of adults and children in the US population

International Journal of Obesity, 24 (2000), pp. 807-818

Godin and Shephard, 1985

G. Godin, R.J. Shephard

A simple method to assess exercise behavior in the community

Canadian Journal of Sport Sciences, 10 (1985), pp. 141-146

Heyward and Wagner, 2004

V.H. Heyward, D.R. Wagner

Applied body composite assessment

Human Kinetics, Champaign, IL (2004) 
Kiefer et al., 2005

I. Kiefer, T. Rathmanner, M. Kunze

Eating and dieting differences in men and women

Journal of Men's Health and Gender, 2 (2005), pp. 194-201

Low, 2009

Y.L. Low

Food diary from the GUSTO birth cohort study

Unpublished Instrument (2009)

Low and Lai, 2010

Y.L. Low, Z.X. Lai

Food diary interview guide

Unpublished Instrument (2010)

Lowe et al., 2006

M.R. Lowe, R.A. Annunziato, J.T. Markowitz, E. Didie, D.L. Bellace, L. Riddell, et al. Multiple types of dieting prospectively predict weight gain during the freshman year of college

Appetite, 47 (2006), pp. 83-90

Marfell-Jones et al., 2006

M. Marfell-Jones, T. Olds, A. Stewart, J.E.L. Carter

International standards for anthropometric assessments

The International Society for the Advancement of Kinanthropometry, Potchefstroom, South

Africa (2006)

Montani et al., 2006

J.P. Montani, A.K. Viecelli, A. Prevot, A.G. Dulloo

Weight cycling during growth and beyond as a risk factor for later cardiovascular diseases. The 'repeated overshoot' theory

International Journal of Obesity, 30 (2006), pp. S58-S66

Muraven and Baumeister, 2000

M. Muraven, R.F. Baumeister

Self-regulation and depletion of limited resources. Does self-control resemble a muscle?

Psychological Bulletin, 126 (2000), pp. 247-259

Norton and Olds, 2002

K. Norton, T. Olds

Anthropometrica 
UNSW Press, Sydney (2002)

Sonnentag and Jelden, 2009

S. Sonnentag, S. Jelden

Job stressors and the pursuit of sport activities. A day-level perspective

Journal of Occupational Health Psychology, 14 (2009), pp. 165-181

Stein and Colditz, 2004

C.J. Stein, G.A. Colditz

The epidemic of obesity

Journal of Clinical Endocrinology \& Metabolism, 89 (2004), pp. 2522-2525

Stice et al., 2004

E. Stice, M. Fisher, M.R. Lowe

Are dietary restraint scales valid measures of acute dietary restriction? Unobtrusive observational data suggest not

Psychological Assessment, 16 (2004), pp. 51-59

van Strien et al., 2006

T. van Strien, R.C.M.E. Engels, W. van Staveren, C.P. Herman

The validity of dietary restraint scales. Comment on Stice et al. (2004)

Psychological Assessment, 18 (2006), pp. 89-94

van Strien et al., 1986

T. van Strien, J.E.R. Frijters, G.P.A. Bergers, P.B. Defares

The Dutch Behavior Questionnaire for assessment of restrained, emotional and external eating behavior

International Journal of Eating Disorders, 5 (1986), pp. 295-315

Zellner et al., 2006

D.A. Zellner, S. Loaiza, Z. Gonzalez, J. Pita, J. Morales, D. Pecora, A. Wolf

Food selection changes under stress

Physiology and Behavior, 87 (2006), pp. 789-793 